\title{
A Damage-Based Maintenance and Repair Optimization Method Using Linear Programming for Bridges
}

\author{
Faraz Masoumi and Ferhat Akgül
}

\begin{abstract}
Maintenance, repair and replacement actions for bridges become necessary due to aging of these structures in time. Apart from heavy traffic loading, the most prominent cause of bridge aging is material deterioration in time due to environmental factors. In the long term, the bridge engineering is expected to gradually focus more on the inevitable stage of maintaining the existing bridges than the stage of constructing new bridges. During the past decades, a variety of bridge maintenance management methodologies have been developed to cost-effectively allocate limited budgets to deteriorating highway bridges. A major objective of a BMS (Bridge Management System) is to assist bridge administrations in making consistent and cost-effective decisions related to bridge activities on a system-wide basis. This paper presents a linear programming optimization technique for the selection of bridge element maintenance and repair actions established as part of a BMS developed in Turkey.
\end{abstract}

Index Terms-Bridges, bridge management systems, deterioration, inspection, maintenance, optimization, repair.

\section{INTRODUCTION}

The highway networks of most developed countries are completed or close to completion and bridge maintenance costs have increased to the point where it is now cheaper to build new bridges than to maintain damaged bridges. However, despite this fact, engineers face a decrease in the need for new bridges and, on the other hand, a significant increase in the number of bridges that should be repaired.

In Turkey, a large part of existing bridges must be maintained and repaired properly before they become deficient in the near future. A report has indicated that approximately $50 \%$ of bridges in Turkey are 50 years of age or older. Therefore, the development of a comprehensive bridge management system for existing bridges is essential. Such a system should enable not only the evaluation of bridge performance, but also the suggestion of rehabilitation strategy which takes into account the limited funds that are available for bridge maintenance and repair. Annual maintenance and repair budget for country's bridges and tunnels is around \$11 Million for 2012, \$5.5 Million of which is for bridges. The budget for new bridge construction is, however, over \$100 Million. The case of inadequate maintenance budget and a substantially high new construction budget is the case for most developing countries.

This paper presents a linear programming optimization

Manuscript received August 6, 2012; revised September 10, 2012.

The authors are with the Engineering Sciences Department, Middle East Technical University, 06531, Ankara, Turkey (e-mail: e168221 @ metu.edu.t; akgul@metu.edu.tr). technique for the selection of bridge maintenance and repair actions established as part of a BMS developed in Turkey. The developed method includes systems of bridges with different numbers of elements where each element may be in one of the four condition states based on the results of an inspection. In this approach, each element is assigned its characteristic damage types and each damage type is assigned its related repair actions.

\section{Condition State Model}

The condition state is the best description of the current status of a bridge or element in terms of deterioration, necessitates actions for improvement of the system. Categories of indices representing different levels of deterioration are used in literature as the condition state models for bridge elements. However, general definitions of condition states causes many difficulties in estimation of parameters like transition probability matrices, maintenance costs and the condition states of bridge elements based on inspection, because the estimated damage level for a bridge has different meanings for elements of that bridge; for example, in a bridge with condition state of 3 , it is possible that the girder be in level 2 and the column be in level 4 or the girder be in level 4 and column be in level 1 . The cost of necessary actions and the condition states of future inspections for these two cases will be so different. In literature, there are plans that analyze the elements of bridges separately, but the same problems also exist in these models. Because of these reasons, in this project the condition states are based on the damage types and the linear programming is solved for possible damage types of elements of each bridge. Such a detailed definition of condition states in the literature does not exist and in this sense the model used in this study will be a pioneer. For each element of a bridge, four condition states are defined, $1=$ No damage or very small damage, $2=$ Small damage, $3=$ High level of damage and $4=$ Critical damage, and the condition state of each bridge is estimated from the condition states of its elements.

For solving the Linear Programming, the optimization process is performed at the level of damage types. Each bridge consists of elements, each with a different number of damage types, and for each damage type, there two actions consisting of "repair" and "no repair". Fig.1 shows two bridges (or systems of elements). The first bridge (Fig. 1(a)) has one element which has three damage types and the second bridge (Fig. 1(b)) has two elements where the first element has three damage types and the second element has two damage types. For each damage type, possible actions will be $\mathrm{a}=0$ (Do nothing action) and $\mathrm{a}=1$ (Maintenance or 
repair action), and the action set has only two elements. The replacement action will be applied when the total cost of maintenance in an element exceeds a predefined percentage of replacement cost.

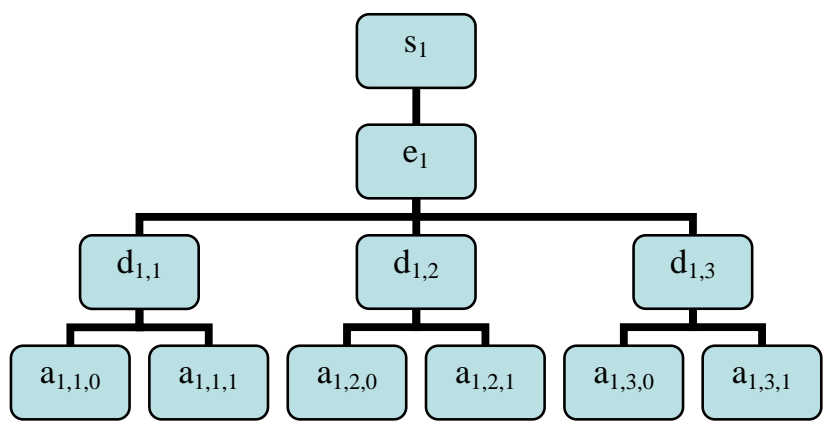

(a)

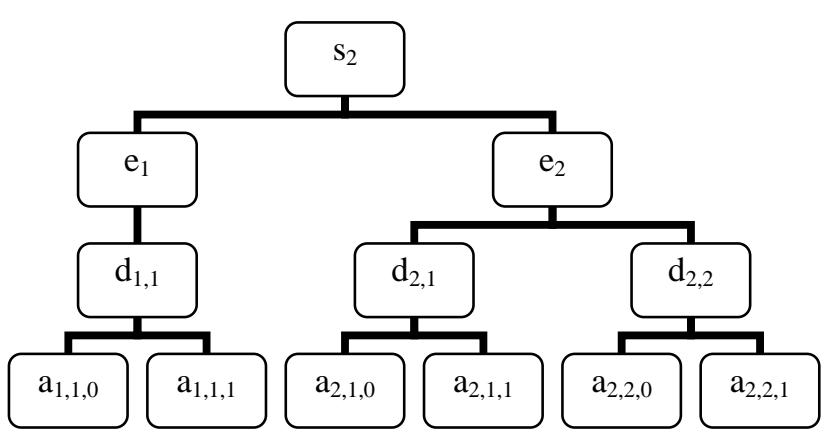

(b)

Fig. 1. Two systems with different number of elements and damage types.

\section{ESTIMATION OF TRANSITION PROBABILITY MATRICES FOR EACH ACTION}

The developed model is based on the Markov Processes reduced to linear programming. In this model, however, for each type of damage type, for the linear programming problem, $P(a)=\left\lfloor p_{i j}(a)\right\rfloor$ matrices for $a=0,1$ actions are estimated. $\mathrm{P}(0)$ is the matrix of transition probabilities of changing the condition states of an element if do nothing action is applied for a damage type and $\mathrm{P}(1)$ is the matrix of probabilities of changing the condition states of an element if an action is applied for a damage type. To make the computations simple, an assumption is made stating that the bridge condition state will not drop by more than one state in a single year and will not improve by more than one state if a maintenance or repair action is applied. The estimated matrices have the following forms:

$$
\begin{gathered}
\mathrm{P}(0)=\left[\begin{array}{cccc}
\mathrm{p}_{1} & 1-\mathrm{p}_{1} & 0 & 0 \\
0 & \mathrm{p}_{2} & 1-\mathrm{p}_{2} & 0 \\
0 & 0 & \mathrm{p}_{3} & 1-\mathrm{p}_{3} \\
0 & 0 & 0 & 1
\end{array}\right] \\
\mathrm{P}(1)=\left[\begin{array}{cccc}
\mathrm{r}_{1} & 1-\mathrm{r}_{1} & 0 & 0 \\
\mathrm{q}_{2} & \mathrm{r}_{2} & 1-\mathrm{q}_{2}-\mathrm{r}_{2} & 0 \\
0 & \mathrm{q}_{3} & \mathrm{r}_{3} & 1-\mathrm{q}_{3}-\mathrm{r}_{3} \\
0 & 0 & \mathrm{q}_{4} & 1-\mathrm{q}_{4}
\end{array}\right]
\end{gathered}
$$

For the estimation of transition probability matrices, the so-called deterioration model is used. Deterioration model is the relationship between bridge condition state and bridge age, which reflects the level of service of a bridge, and therefore, can be used as a measure of effectiveness. The element age is represented by $\mathrm{T}$ and the condition state by $\mathrm{Y}(\mathrm{T})$. For instance, if the condition state of the element $\mathrm{E}_{1}$ of bridge $\mathrm{B}_{1}$ in 2010 was 2 at the age of $\mathrm{T}=24$ then $Y(24)=2$.

One of the properties of Markov chains is that the transition probability matrices of a two or more-year period will be equal to a power of the transition probability matrix of the one-year period as given in Eq. 3 below.

$$
P\left\{X_{n+k}=j \text { if } A_{n}=0, \ldots, A_{n+k=0}\right\}=\left[P(0)^{k}\right]_{i j}
$$

Using this equation, the condition state of an element which is in state $\mathrm{i}$ in time $\mathrm{n}$ after $\mathrm{k}$ periods of time will be:

$$
\begin{aligned}
& E_{i}^{k}= \\
& \sum_{j=1}^{M}\left\{j P X_{n+k}=j \text { if } X_{n}=i, A_{n}=0, A_{n+1}=0, . ., A_{n+k}=0\right\}
\end{aligned}
$$

So, the elements of the matrix $\mathrm{P}(0)$ for each element can be expressed as unknowns. To estimate the transition matrix probabilities, the following non-linear programming objective function is formulated and solved:

$$
\begin{aligned}
& \min \sum_{t=1}^{10}\left|Y(t)-E_{p}^{t}\right| \\
& \text { subject to } 0 \leq p_{i} \leq 1, i=1,2,3,4
\end{aligned}
$$

The objective function is to minimize the absolute distance between the actual bridge condition state at a certain age, and the predicted bridge condition for the corresponding age generated by the Markov chain with probabilities obtained from the non-linear programming. The LINGO software is used for solving this nonlinear programming and, as a result, the transition probability matrix for the do-nothing action $\mathrm{P}(0)$ is estimated. The action matrix $\mathrm{P}(1)$ is deduced from $\mathrm{P}(0)$ matrices based on expert knowledge. The experts were asked to state the possibilities of improvements for different types of materials (concrete, steel, stone, etc.) and this information was used to estimate the matrices.

\section{OPtimizATION MAINTENANCE AND REPAiR ACTIONS}

Markov decision process is used for determination of maintenance and repair actions which are random [1], [2]. The condition state in time $\mathrm{n}$ is $\mathrm{X}_{\mathrm{n}}$, and all condition states compose condition state set of $\mathrm{S}=\{1,2, \ldots, \mathrm{M}\}$ with a finite number of elements. The action in time $n$ is $A_{n}$ and all actions also compose actions set of $\mathrm{A}=\{1,2, \ldots, \mathrm{D}\}$ with a finite number of elements. $C_{i a}$ is the cost of the action a 
taken at condition state $\mathrm{i}$ and for each damage type is estimated by using unit costs. Probability of changing the condition state of an element from state $i$ to state $\mathrm{j}$ in time $\mathrm{n}+1$ if action a is taken is $p_{i j}(a)=P\left\{X_{n+1}=j\right.$ if $\left.X_{n}=i, A_{n}=a\right\}$. The policy of minimization of expected cost in the long term is solved by linear programming (LP) as shown below [3]:

$$
\begin{gathered}
\min \sum_{i=1}^{M} \sum_{a=1}^{D} C_{i a} X_{i a} \\
\sum_{a=1}^{D} X_{j a}=\sum_{i=1}^{M} \sum_{a=1}^{D} X_{i a} p_{i j}(a) j=1,2, \ldots, M \\
\sum_{i=1}^{M} \sum_{a=1}^{D} X_{i a}=1 \quad j=1,2, \ldots, M, a=1,2, \ldots, D \\
\mathrm{X}_{\mathrm{ia}} \geq 0
\end{gathered}
$$

The obtained result from this model is the steady state probabilities in the long term if the element is initially in state Iand action $\mathrm{a}$ is applied.

The optimal solution will always be unique for each state. In other words, for state $\mathrm{i}, \mathrm{X}_{\mathrm{ib}} \geq 0$ will be positive only for one action, say $b, X_{i b} \geq 0$, and for all other actions, $X_{i a}$ will be zero. This means that, in order to obtain the optimal policy, if the system is in state $\mathrm{i}$, action $\mathrm{b}$ should be applied.

\section{Linear Programming SOlUtion}

Linear programming can be solved for each damage type of the elements of a bridge. The result obtained from linear programming is the optimal policy defining the actions to be applied to the damage types of the elements. For instance, if the condition state of damage type of $D_{1}$ in element $e_{1}$ of bridge $S_{1}$ is 3 and the optimal policy for that damage type is obtained as shown in Table I, then the necessary action will be "1-Maintenance or repair action"

TABLE I: OPTIMAL POLICY FOR A DAMAGE TYPE FOR EACH CONDITION STATE.

\begin{tabular}{cl}
\hline State $i$ & \multicolumn{1}{c}{ Action $a$} \\
\hline 1 & 0 - Do nothing action \\
2 & 0 - Do nothing action \\
3 & 1 - Maintenance or repair action \\
4 & 1 - Maintenance or repair action \\
\hline
\end{tabular}

\section{PRIORITIZATION OF BRIDGES}

The maintenance expenditures should not exceed the yearly allocated budget. For this reason, maintenance plans should be prepared and bridges should be evaluated according to various criteria. Some of these criteria in literature include but not limited to the condition state of bridge, average daily traffic, maintenance cost, and user cost [4],[ 5]. The first three criteria are used in this study and the bridges are prioritized based on these criteria. The three criteria does not have the same importance, therefore a multi-objective decision making solution is used. For each bridge, a multi objective index (MOI) is calculated using the following formula:

$$
\begin{aligned}
& \operatorname{MOI}(\mathrm{x})= \\
& \sqrt{\left(\mathrm{W}_{\mathrm{C}} \frac{\left|\mathrm{C}^{*}-\mathrm{C}_{\mathrm{b}}\right|}{\left|\mathrm{C}^{*}-\mathrm{C}_{*}\right|}\right)^{2}+\left(\mathrm{W}_{\mathrm{A}} \frac{\left|\mathrm{A}^{*}-\mathrm{A}_{\mathrm{b}}\right|}{\left|\mathrm{A}^{*}-\mathrm{A}_{*}\right|}\right)^{2}+\left(\mathrm{W}_{\mathrm{M}} \frac{\left|\mathrm{M}^{*}-\mathrm{M}_{\mathrm{b}}\right|}{\left|\mathrm{M}^{*}-\mathrm{M}_{*}\right|}\right)^{2}}
\end{aligned}
$$

where:

$\mathrm{W}_{\mathrm{C}}$ : Weight for the condition state, $\mathrm{W}_{\mathrm{A}}$ : Weight for the average daily traffic, $\mathrm{W}_{\mathrm{M}}$ : Weight for the maintenance cost $\mathrm{C}^{*}$ : Maximum of condition states of all bridges, $\mathrm{C}_{*}$ : minimum of condition states of all bridges, $\mathrm{C}_{\mathrm{b}}$ :condition state of bridge $b$

$\mathrm{A}^{*}$ : Maximum of average daily traffic values of all bridges, $\mathrm{A}_{*}$ : minimum of average daily traffic values of all bridges, $A_{b}$ : average daily traffic value of bridge $b$

$\mathrm{M}^{*}$ : Maximum of maintenance costs of all bridges, $\mathrm{M}_{*}$ : minimum of maintenance costs of all bridges, $M_{b}$ : maintenance cost of bridge $b$

The sum of the weights should be equal to one, i.e. $\mathrm{W}_{\mathrm{C}}+\mathrm{W}_{\mathrm{A}}+\mathrm{W}_{\mathrm{M}}=1$

\section{CONCLUSION}

A maintenance and repair optimization routine is developed for a bridge management system using Fortran language and it is applied to determine the optimal maintenance and repair policies for 200 existing bridges. The novel feature of the optimization method is that it is based on damage types of elements. At first, the input file for the bridges is automatically generated based on based on inspection and cost data. The input file includes transition probability matrices and cost matrices of all damage types of all elements of the bridges. Then, the optimization program is solved for the subject bridges. The output file of optimization program is used in prioritization (ranking) module. The prioritization module determines which damage types of elements of bridges should be repaired based on an available budget.

\section{REFERENCES}

[1] A. Agrawal, A. Kawaguchi and Z. Chen, "Deterioration Rates of Typical Bridge Elements in New York," Journal of Bridge Engineering vol. 15, pp. 419-429, 2010.

[2] Y. Kuo, "Optimal adaptive control policy for joint machine maintenance and product quality control," European Journal of Operational Research vol. 171, pp. 586-597, 2006.

[3] F. S. Hillier and G. J Lieberman, Introduction to Operations Research, McGraw-Hill, 2010.

[4] Z. Lounis and D. J. Vanier, "A Multiobjective and Stochastic System for Building Maintenance Management," Computer-Aided Civil and Infrastructure Engineering vol. 15, pp. 320-329, 2000. 
[5] Z. Lounis, "Risk-based maintenance optimization of bridge structures," National Research Council, Canada, NRCC-47063, 2004.

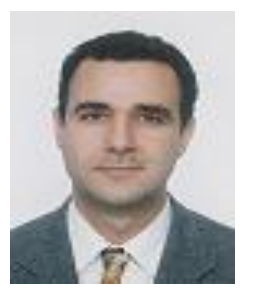

Ferhat Akgül received his B.S. degree in Civil Engineering from Middle East Technical University, Turkey, in 1987, and M.S. in Civil Engineering; Structural Mechanics form University of New Mexico, Albuquerque, New Mexico, in 1989 and Ph.D. degrees in Civil Engineering, Structural Mechanics from University of Colorado at Boulder, Colorado, in 2002. He is currently an assistant professor at the Engineering Sciences Department, the Middle East Technical University. He has over 10 years of teaching experience and has published many papers in national and international journals. His research interest includes Structural Reliability, Lifetime
Performance Prediction of Bridges and Simulation Methods. Dr. Akgül is a member of IABMAS, International Association for Bridge Maintenance and Safety.

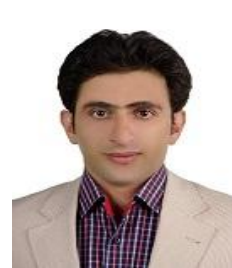

Faraz Masoumi received his B.S. degree in Civil Engineering from Azarbaijan University of Tarbiat Moallem, Iran, in 2005 and M.S. in Civil Engineering, Structure from Islamic Azad University, Maragheh Branch, in 2007. He is currently Ph.D. Candidate in Engineering Sciences Department Middle East Technical University, Ankara, Turkey. 